\section{Immune response of neonates to oral poliomyelitis vaccine}

In the conventional schedule of immunisation infants are given three doses of oral poliomyelitis vaccine starting at, or after, 6 to 8 weeks of age. In the new pulse immunisation strategy three doses of oral poliomyelitis vaccine are given in annual cycles to children under 24 months of age. ${ }^{1}$ I carried out a study to see whether a lower age limit could be established in this programme. Neonates were given one dose of oral poliomyelitis vaccine followed by second and third doses at intervals of four weeks. The seroconversion response in the neonates was as good as the response in older infants, indicating that immunisation may be started in the neonatal period.

\section{Patients, methods, and results}

I studied 180 neonates and infants with parental consent. The first dose of oral poliomyelitis vaccine was given to groups of 30 infants at $7,14,21$, 28,35 , and 42 days ( $t$ one day) of age. Second and third doses were given at intervals of four weeks. The vaccine was tested and found to be fully potent. At least 30 minutes was allowed between administration of the vaccine and breast feeding. Capillary blood was collected immediately before giving the first dose of the vaccine and four weeks after the last dose. The second sample of blood was collected from only 139 infants.

The paired serum samples were tested together in doubling dilutions from $1 / 8$ to $1 / 1024$ for poliovirus neutralising antibodies as described previously. ${ }^{2}$ The second serum sample was collected 12 weeks after the first. As the half life of maternal antibody in the infant's circulation is less than four weeks the persisting maternal antibody titre in the second serum sample would be lower than that in the first by a factor of eight or more. A fourfold or higher titre than the expected residual maternal antibody titre in the second serum sample was taken to indicate the infant's immune response (seroconversion) to the three doses of oral poliomyelitis vaccine. ${ }^{2} 3$

The table shows the frequency of the antibody response in the neonates and infants to three doses of poliomyelitis vaccine according to age at the start of immunisation. It also compares the antibody response of infants aged from 6 to 20 weeks with maternal antibody with that of infants aged from 6 to 51 weeks without maternal antibody from a previous study. ${ }^{2}$ The seroconversion response to the three types of poliovirus in infants receiving their first dose during the neonatal period was no less than that in older infants.

Antibody response in neonates and infants to three doses of oral poliomyelitis vaccine according to age at which immunisation was started

\begin{tabular}{|c|c|c|c|c|c|}
\hline \multirow{2}{*}{$\begin{array}{c}\text { Age } \\
\text { (weeks)* }\end{array}$} & \multirow{2}{*}{$\mathrm{n}$} & \multicolumn{3}{|c|}{ Seroconversion response $\left({ }_{0}^{0}\right)$ to: } & \multirow{2}{*}{$\begin{array}{c}\text { Mean } \\
\text { seroconversion } \\
\text { response } \\
\left(0_{0}\right)\end{array}$} \\
\hline & & Type 1 & Type 2 & Type 3 & \\
\hline $\begin{array}{l}1 \\
2 \\
3 \\
4 \\
5 \\
6\end{array}$ & $\begin{array}{l}23 \\
30 \\
25 \\
26 \\
19 \\
16\end{array}$ & $\begin{array}{l}83 \\
80 \\
64 \\
90 \\
47 \\
69\end{array}$ & $\begin{array}{l}83 \\
90 \\
96 \\
95 \\
68 \\
81\end{array}$ & $\begin{array}{l}78 \\
70 \\
56 \\
65 \\
42 \\
63\end{array}$ & $\begin{array}{l}81 \\
80 \\
72 \\
83 \\
53 \\
71\end{array}$ \\
\hline $1-6$ & 139 & 73 & 87 & 63 & 75 \\
\hline $\begin{array}{l}6-20(\text { ref } 2) \\
6-51(\text { ref } 2)\end{array}$ & $\begin{array}{l}86 \\
61\end{array}$ & $\begin{array}{l}72 \\
66\end{array}$ & $\begin{array}{l}88 \\
95\end{array}$ & $\begin{array}{l}79 \\
72\end{array}$ & $\begin{array}{l}80 \\
78\end{array}$ \\
\hline
\end{tabular}

*Age when first dose of oral poliomyelitis vaccine given.

\section{Comment}

As the seroconversion response in infants beginning immunisation from 1 to 4 weeks of age was not less than that in older infants, such neonates may be given oral poliomyelitis vaccine either in the conventional schedule of immunisation or in the pulse immunisation strategy. If the five dose schedule of primary immunisation with poliomyelitis vaccine is followed the first two doses may be given as early as 1 and 5 weeks of age followed by three doses of diphtheria pertussis, and tetanus and oral poliomyelitis vaccine at 9, 13, and 17, weeks. ${ }^{4}$ In pulse immunisation the lower age limit for oral poliomyelitis vaccine should be 1 week.

I did not investigate the immune response to oral poliomyelitis vaccine in neonates who were less than 1 week of age. Early investigations on the vaccine showed that there was some inhibition of response in neonates during the first three days of life. ${ }^{3}$ The presence of maternal antibody did not seem to inhibit the infant's ability to produce antibodies to the vaccine. Young infants with maternal antibody have recently been immunised with an aerosolised live measles virus vaccine. ${ }^{5}$ Though passive immunity inhibits the response to parenteral stimulation by live vaccines, it does not seem to prevent local infection by poliovirus in the gut or by measles virus in the respiratory tract and the consequent active immune response.

I thank Mrs Saroja Christopher for technical help. The centre of virology is supported by the Indian Council of Medical Research, New Delhi. 1 John TJ, Steinhoff MC. Appropriate strategy for immunization of children in India. 3. Com $677-83$.

2 John TJ, Devarajan LV, Luther L, et al. Effect of breast-feeding on seroresponse of infants to oral poliovirus vaccination. Pediatrics $1976 ; 57: 47-53$.

3 warren RJ, Lepow ML, Bartsch GE, et al. The relationship of maternal antibody, breast feeding and age to the susceptibility of newborn infants to infection with attenuated polioviruses. Pediatrics $1964 ; 34: 4-13$.

$4 \mathrm{John}$ TJ. Antibody response of infants in tropics to five doses of oral polio vaccine. $B r$ Med F 1976;i:812.

5 Sabin AB, Arechiga AF, de Castro JF, et al. Successful immunization of children with and without maternal antibody by aerosolized measles vaccine. Different results with undiluted human diploid cell and chick embryo fibroblast vaccines. $\mathcal{F} A M A$ 1983;249:2651-62.

(Accepted 26 fuly 1984)

Indian Council of Medical Research Centre of Virology, Christian Medical College Hospital, Vellore 632 004, India

T JACOB JOHN, FRCPED, PHD, professor and programme director

\section{Survival after prolonged cardiac arrest and accidental hypothermia}

Profound accidental hypothermia has been associated with a mortality of up to $80 \%$, but more recent reports indicate much lower mortality provided there is no underlying pathological condition. ${ }^{2}$ Successful resuscitation from deep accidental hypothermia and cardiac arrest of several hours' duration has been reported in several patients without any underlying disease. ${ }^{3}$ Most of these successful resuscitations have entailed the use of extracorporeal circulation. ${ }^{3}$ We report on a patient with accidental hypothermia who was warmed with simpler methods available in most district general hospitals and who survived four hours of cardiac arrest.

\section{Case report}

A thin 27 year old unemployed man was admitted deeply unconscious with no detectable cardiac sounds or pulses and gasping intermittently. Ambulancemen reported that he had been sleeping on a park bench overnight; his wife suspected that he had taken some barbiturates after ingesting alcohol. The doctor in the casualty department immediately passed an endotracheal tube, and the cardiac rhythm changed from slow sinus of 48 beats/minute with $J$ waves to ventricular fibrillation. Full cardiac arrest procedure was begun: he was given sodium bicarbonate, lignocaine, direct current cardioversion, adrenaline and calcium infusions, external cardiac massage, and ventilation with $100 \%$ oxygen. There was no response.

He was transferred to the intensive therapy unit, where he was ventilated with warmed, humidified oxygen and external cardiac massage was continued. Lower oesophageal temperature was $25^{\circ} \mathrm{C}$. Blood samples were taken for estimation of urea, electrolyte, haemoglobin, and barbiturate concentrations and gas tensions; he was given magnesium sulphate $4 \mathrm{~g}$ as cerebral protection. Rewarming continued with gastric lavage with warmed fluid, and a warmed intravenous infusion was set up, the rate of infusion being adjusted according to the central venous pressure. These measures were thought necessary because of the contraction of plasma volume that occurs early on in hypothermia and contributes to the hypotension when vasodilatation occurs on rewarming, especially in the presence of heart failure induced by hypothermia.

Core temperature was $31^{\circ} \mathrm{C}$ four hours later, when defibrillation was successful, and blood pressure was $90 / 60 \mathrm{~mm} \mathrm{Hg}$. In common with other survivors of prolonged cardiac arrest in accidental hypothermia he developed acute pulmonary oedema. This initially responded to simple treatment with diuretics and oxygen, but it recurred and the adult respiratory distress syndrome developed, which together made artificial ventilation necessary for nearly three weeks. Less than a month later, however, he went home fully recovered and with no neurological deficit.

\section{Comment}

Alcohol, hypothermia, and barbiturates are known to prolong survival of neurones in circulatory arrest and probably contributed to the survival of this patient. ${ }^{2}$ Our main reason for presenting this case, however, is to show that good results may be obtained without resorting to extracorporeal circulation. With simple measures and equipment available in most district general hospitals central re- 